Disclosure of Interest: M. Ochiai: None declared, E. Tanaka Consultant for: Abbvie, Eisai Pharmaceutical, Chugai Pharmaceutical, Bristol Myers Squibb, Astellas Pharmaceutical, Pfizer, Takeda Pharmaceutical, and Ayumi Pharmaceutical., Speakers bureau: Abbvie, Eisai Pharmaceutical, Chugai Pharmaceutical, Bristol Myers Squibb, Astellas Pharmaceutical, Pfizer, Takeda Pharmaceutical, and Ayumi Pharmaceutical., E. Inoue: None declared, R. Yamaguchi: None declared, Y. Shimizu: None declared, N. Sugimoto Speakers bureau: Takeda Pharmaceutical and Bristol Myers Squibb., K. Ikari Grant/research support from: Astellas, UCB, Bristol-Meyers, Pfizer, Eisai, Tanabe-Mitsubishi, Chugai, AbbVie, Janssen Pharmaceutical, Otsuka, Kaken, Asahi-Kasei, Hisamitsu and Takeda., Speakers bureau: Astellas, UCB, Bristol-Meyers, Pfizer, Eisai, Tanabe-Mitsubishi, Chugai, AbbVie, Janssen Pharmaceutical, Otsuka, Kaken, Asahi-Kasei, Hisamitsu and Takeda., A. Nakajima Consultant for: Bristol-Meyers, Mitsubishi Tanabe Pharma, Nippon Kayaku Co. Ltd., Novartis Pharma, Pfizer, Siemens Healthcare Diagnostics K.K. and Takeda Pharmaceutical Company, Speakers bureau: Bristol-Meyers, Mitsubishi Tanabe Pharma, Nippon Kayaku Co. Ltd., Novartis Pharma, Pfizer, Siemens Healthcare Diagnostics K.K. and Takeda Pharmaceutical Company, A. Taniguchi Grant/research support from: AbbVie, Eisai, Takeda, TanabeMitsubishi, Teijin Pharma, Pfizer., Speakers bureau: AbbVie, Eisai, Takeda, Tanabe-Mitsubishi, Teijin Pharma, Pfizer., H. Yamanaka Grant/research support from: MSD, Ayumi, AbbVie, Eisai, Ono, Astellas, Daiichi-Sankyo, Taisyo-Toyama, Takeda, Tanabe-Mitsubishi, Chugai, Teijin Pharma, Torii, Nippon Shinyaku, Pfizer. UCB. Nippon Kayaku, YL biologics, Bayer and Bristol-Meyers., Consultant for: MSD, Ayumi, AbbVie, Eisai, Ono, Astellas, Daiichi-Sankyo, Taisyo-Toyama, Takeda, Tanabe-Mitsubishi, Chugai, Teijin Pharma, Torii, Nippon Shinyaku, Pfizer. UCB. Nippon Kayaku, YL biologics, Bayer and Bristol-Meyers., Speakers bureau: MSD, Ayumi, AbbVie, Eisai, Ono, Astellas, Daiichi-Sankyo, Taisyo-Toyama, Takeda, Tanabe-Mitsubishi, Chugai, Teijin Pharma, Torii, Nippon Shinyaku, Pfizer. UCB. Nippon Kayaku, YL biologics, Bayer and Bristol-Meyers.

DOI: 10.1136/annrheumdis-2017-eular.1902

\section{FRI0118 CLINICAL UTILITY OF AREA-UNDER-CURVE (AUC) OF PATIENT-DERIVED DISEASE ACTIVITY SCORE (PDAS2) BETWEEN CLINIC VISITS ON REMISSION, FLARE UP AND RHEUMATOLOGIST'S DECISION TO ESCALATE ANTI-RHEUMATIC DRUGS}

M.-H.A. Leung ${ }^{1}$, E. Choy ${ }^{2}$, C.S. Lau ${ }^{3} .{ }^{1}$ Department of Medicine, Queen Elizabeth Hospital, Kowloon, Hong Kong: ${ }^{2}$ Arthritis Research UK, Health and Care Research Wales CREATE Centre, Institute of Infection and Immunity, Cardiff University, Cardiff, United Kingdom; ${ }^{3}$ Department of Medicine, LKS Faculty of Medicine, University of Hong Kong, Hong Kong, Hong Kong

Background: The standard of care in RA is treat-to-target of remission or low disease activity state (LDAS). Integral to this is the regular assessment of disease activity. Patient-derived Disease Activity Score 2 (PDAS2) was developed to allow RA patients to self-assess. Validation, corresponding disease activity statuses cut-points and response criteria had been published. PDAS2 scores $<3.8,3.8-4.5,4.6-5.0,>5.0$ correspond to remission, LDAS, moderate and high disease activities respectively. PDAS2 can be recorded by patients in-between clinic visits.

Objectives: To explore the clinical utility of PDAS2 on remission, flare and need of drug adjustment

Methods: A cohort of 100 consecutive RA patients was recruited to complete PDAS2 score at home fortnightly in between two consecutive rheumatology clinics. Patients would return the forms when they attended the second clinic. Rheumatologists adjusted treatment according to disease activity while blinded to the scores of PDAS2 recorded at home. AUC of PDAS2 was calculated from the mean of (PDAS2 score multiplied by the time interval between scores). They were compared with disease activity at the first and second visits. The change of PDAS2 score for those patients having SDAl flare-up (from remission/LDAS to moderate/high disease activity) was compared to those didn't flare-up using unpaired T-test. Receiver Operator Characteristic curve was used to determine the cut-point for AUC-PDAS2 increment to predict flare-up and the cut-point of PDAS2 score for rheumatologists to escalate anti-rheumatic drugs.

Results: Mean age of the cohort was 60 years, mean RA duration 14 years, $90 \%$ female, $71 \%$ sero-positive and $48 \%$ in remission/low disease activity. 89 patients $(89 \%)$ returned written questionnaires which were done $7.8 \pm 3.5$ times (mean \pm standard deviation) (range 1-16) for a follow-up interval of $17.5 \pm 9.4$ weeks (range 3.9-60.3). Disease activities in first and second visits are shown in Table. Remission: For the 14 patients in SDAI remission in both visits, 13/14 were in AUC-PDAS2 remission, and 1/14 in LDAS. There were 47,45 and 37 out of 89 patients in SDAI, CDAI and DAS28 remission/LDAS respectively - they were all in AUC-PDAS2 remission/LDAS. Flare-up: There were 10/89 patients in SDAI remission/LDAS in first visit and moderate/high activity in second visit. Their AUC-PDAS2 score rose by $0.33 \pm 0.35$ points compared to $0.01 \pm 0.32$ who had no flare-up $(p=0.002)$. ROC curve AUC was 0.80 (95\% Cl: $0.64,0.95)(p=0.002)$, with optimal cut-point at increment of AUC-PDAS2 score by 0.11 to predict flare, sensitivity and specificity both being $80 \%$. Moreover, rheumatologists decided to escalate anti-rheumatic drugs in $15 / 89$ patients. ROC curve AUC was $0.71(95 \%$ $\mathrm{Cl}: 0.56,0.86)(\mathrm{p}=0.01)$, with optimal cut-point at PDAS2 score 4.33 to predict the need of escalating anti-rheumatic drugs, sensitivity being $60 \%$ and specificity $77 \%$.

\begin{tabular}{lllllll} 
& & \multicolumn{2}{c}{ First visit } & & \multicolumn{2}{c}{ Second visit } \\
SDAI & $\mathrm{N} \quad(\%)$ & AUC-PDAS2 (SD) & $\mathrm{N}$ & (\%) & AUC-PDAS2 (SD) \\
\cline { 3 - 6 } & Remission & $22(24.7 \%)$ & $3.23(0.40)$ & $22(24.7 \%)$ & $3.22(0.43)$ \\
& LDAS & $36(40.4 \%)$ & $3.72(0.52)$ & $35(39.5 \%)$ & $3.80(0.55)$ \\
& Moderate & $29(32.6 \%)$ & $4.50(0.60)$ & $30(33.7 \%)$ & $4.40(0.66)$ \\
& High & $2(2.2 \%)$ & $5.26(1.08)$ & $2(2.2 \%)$ & $5.21(1.15)$
\end{tabular}

Table. Simplified Disease Activity Index (SDAI) disease activities of RA patients ( $N=89$ ) in first and second visits, and corresponding mean area-under-curve of Patient Disease Activity Score 2 (AUC-PDAS2)

Conclusions: PDAS2 scoring by patients in-between follow-up is feasible and useful in reassuring RA patients kept in remission/LDAS, informing a potential flare from previous remission/LDAS state, and predicting rheumatologists' decision to escalate anti-rheumatic drugs. AUC-PDAS2 concept is useful in development of smartphone application for patient use.

References:

[1] A\&R 2008 Feb 15;59(2):192-9.

[2] Rheumatology $2016 \mathrm{Nov} ; 55(11): 1954-8$

Disclosure of Interest: None declared

DOI: 10.1136/annrheumdis-2017-eular.2788

\section{FRI0119 A PROPOSAL FOR A SDAI, CDAI, AND RAPID3-BASED DEFINITION OF MINIMAL DISEASE ACTIVITY FOR USE IN ROUTINE CARE OF RHEUMATOID ARTHRITIS: RESULTS FROM A JAPANESE NATIONAL DATABASE}

N. Yokogawa ${ }^{1}$, A. Komiya ${ }^{2}$, K. Shimada ${ }^{1}$, S. Sugii ${ }^{1}$, J. Nishino ${ }^{3}$, S. Tohma ${ }^{4}$. ${ }^{1}$ Department of Rheumatic Diseases, Tokyo Metropolitan Tama Medical Center, Tokyo; ${ }^{2}$ Department of Clinical Laboratory, Sagamihara National Hospital, National Hospital Organization, Kanagawa; ${ }^{3}$ Department of Orthopaedic Surgery and Spinal Surgery, he University of Tokyo, Tokyo; ${ }^{4}$ Clinical Research Center for Allergy and Rheumatology, Sagamihara National Hospital, National Hospital Organization, Kanagawa, Japan

Background: The OMERACT group proposed minimal disease activity (MDA) as a treatment target, given the current treatment possibilities and limitations. Whereas a Disease Activity Score 28 (DAS28)-based definition of MDA has been proposed $^{1}$, definitions based on the Simplified Disease Activity Index (SDAI), Clinical Disease Activity Index (CDAI), and Routine Assessment of Patient Index Data 3 (RAPID3) have not, despite the increasing use of these indices.

Objectives: To define SDAI, CDAI, and RAPID3-based definition of MDA for use in routine care.

Methods: We analyzed 15,101 patients registered in the Japanese National Database (NinJa 2015). As the OMERACT group proposed, patients with tender joint count (TJC) of 0 , swollen joint count (SJC) of 0 , and erythrocyte sedimentation rate $(E S R) \leq 10 \mathrm{~mm} /$ hour or patients with five of the following seven criteria, namely, pain $\leq 2, \mathrm{SJC} \leq 1, \mathrm{TJC} \leq 1, \mathrm{HAQ} \leq 0.5$, physician's global $\leq 1.5$, patient's global $\leq 2$, and ESR $\leq 20$, were considered to be in MDA. ${ }^{1}$ The ROC curve was used to obtain the best cut-off points for the SDAI, CDAI, and RAPID3-based definitions of MDA, which emerged as good predictors of MDA as defined by the core dataset. To compare the usefulness of the indices, the interclass correlation of MDA in DAS28, SDAI/CDAI, and RAPID3 was compared to that of low disease activity (LDA).

Results: $57.6 \%$ of patients $(5,629 / 9,767)$ were categorized as having MDA, and $29 \%$ of patients $(4,003 / 13,781)$ were categorized as Boolean remissions. In the ROC analysis, the area under the curve for DAS28, SDAI, CDAI, and RAPID3 was $0.911,0.955,0.953$, and 0.930 , respectively. Based on the Youden index, SDAI $\leq 5.3$, CDAI $\leq 4.8$, and RAPID3 $\leq 5$ were defined as SDAI, CDAI, and RAPID3based MDA, respectively. The sensitivity and specificity of the DAS28, SDAI, CDAI, and RAPID3-based definitions were higher than those of the DAS28-based definition, with a sensitivity of $81.5 \%, 89.2 \%, 88.8 \%$, and $90.0 \%$, respectively, and a specificity of $83.5 \%, 89.4 \%, 89.9 \%$, and $88.4 \%$, respectively. Each index-based definition of MDA showed better interclass correlation than that of LA; DAS28 vs CDAI/SDAI: MDA of $0.643 / 0.662$ and LDA of $0.540 / 0.540$; DAS28 vs RAPID3: MDA of 0.541 and LDA of 0.482 ; CDAI/SDAI vs RAPID3: MDA of $0.677 / 0.671$ and LDA of $0.433 / 0.425$.

Conclusions: SDAI $\leq 5.3, \mathrm{CDAI} \leq 4.8$, and RAPID3 $\leq 5$, values two points higher than each remission criterion, may provide a more stringent therapeutic goal than LDA in clinical practice.

References:

[1] Wells GA, et al. Minimal disease activity for rheumatoid arthritis: a preliminary definition. J Rheumatol 2005;32:2016-24.

Acknowledgements: Supported in part by a Health and Labor Sciences Research Grant from the Ministry of Health, Labor, and Welfare of Japan.

Disclosure of Interest: None declared

DOI: 10.1136/annrheumdis-2017-eular.3339 